University of Nebraska - Lincoln

DigitalCommons@University of Nebraska - Lincoln

November 1993

\title{
Brillouin-scattering study of the elastic constants of phenothiazine through the phase transition
}

\author{
J. Sartwell \\ University of Nebraska - Lincoln \\ Craig J. Eckhardt \\ University of Nebraska - Lincoln, ceckhardt1@unl.edu
}

Follow this and additional works at: https://digitalcommons.unl.edu/chemistryeckhardt

Part of the Chemistry Commons

Sartwell, J. and Eckhardt, Craig J., "Brillouin-scattering study of the elastic constants of phenothiazine through the phase transition" (1993). Craig J. Eckhardt Publications. 16.

https://digitalcommons.unl.edu/chemistryeckhardt/16

This Article is brought to you for free and open access by the Published Research - Department of Chemistry at DigitalCommons@University of Nebraska - Lincoln. It has been accepted for inclusion in Craig J. Eckhardt Publications by an authorized administrator of DigitalCommons@University of Nebraska - Lincoln. 


\title{
Brillouin-scattering study of the elastic constants of phenothiazine through the phase transition
}

\author{
J. Sartwell* and C. J. Eckhardt ${ }^{\dagger}$ \\ Department of Chemistry, University of Nebraska, Lincoln, Nebraska 68588-0304
}

(Received 21 April 1993)

\begin{abstract}
It has been proposed that single crystals of phenothiazine undergo a proper ferroelastic phase transition: Pnma $\left(D_{2 h}\right) \rightarrow P 2_{1} / c\left(C_{2 h}\right)$. This premise is in conflict with Brillouin-scattering studies from both quasi- and high-symmetry longitudinal and transverse acoustic modes reported here. The $C_{55}$ elastic constant of phenothiazine as an orthorhombic crystal at ambient conditions shows no temperature dependence. The inelastic, light-scattering spectra support an order-disorder phase transition; no significantly unstable acoustic phonons were observed through the phase transition. Laser diffraction from structural domains reveals that the crystal is polysynthetically twinned above and below $T_{c}$. At room temperature, domain walls are made indistinct by disorder but become visible as the sample is cooled. Stress-strain hysteresis below $T_{c}$ is attributed to a ferrobielastic effect.
\end{abstract}

\section{INTRODUCTION}

Phenothiazine is a nonplanar, tricyclic, aromatic compound with the structure shown in Fig. 1. In crystalline form, it is known to undergo a structural phase transition (SPT) that results in the generation of visible striae within the crystal. ${ }^{1}$ Speculations on the microscopic origins of the SPT and the formation of such striae have become a significant area of controversy. Interest in phenothiazine was stimulated by the conjecture of Nakayama and coworkers ${ }^{1-3}$ that, upon cooling, a strain forms spontaneously within the crystal as the result of a structural change from an orthorhombic to a monoclinic lattice. They also observed that striae arise from lamellar domains of the low-temperature phase of phenothiazine, which can be altered by the application of a small tensile stress. Such domains persist until a different stress is applied. Hysteritic behavior is characteristic of ferroelasticity. Nevertheless, domain formation and even domain switching can be associated with several other phenomena such as order-disorder behavior, polysynthetic twinning and ferrobielasticity. ${ }^{4}$ Ferroelasticity has been observed only in a small number of organic crystals, and, as yet, has not been unequivocally determined for the SPT in phenothiazine.

To date, one ultrasound and a number of Raman and Brillouin studies have been used to examine the role of the acoustic and optical phonons in the SPT of behavior. $^{1-3,5-7}$ Although the evidence is conflicting, instabilities in the $e_{5}$ shear and the totally symmetric $e_{3}$ strain have been associated with the SPT. ${ }^{3,6}$ These shear and strain instabilities are consistent with a true ferroelastic SPT, which requires an orthorhombic to a monoclinic structural transition and the softening of a $B_{2 u}$ acoustic mode. ${ }^{4}$ However, the light-scattering data are not consistent with order-disorder behavior., 6 In addition, recent NMR studies point to a phase transition that is governed by strong rotation-translation coupling rather than just by an overdamping of an acoustic mode. ${ }^{8}$ The low-energy, Raman-active modes display a significant phenothiazine in light of possible ferroelastic

amount of anharmonicity through the phase transition.

Conclusions made from the light-scattering studies are problematic when characteristics of high-purity single crystals of phenothiazine are considered. The reported relative magnitudes of the $C_{11}, C_{22}$, and $C_{33}$ elastic constants are inconsistent with the macroscopic properties of the crystal (the room-temperature unit cell is designated: $c>b>a$ following the convention of Nakayama and Ishii $^{2}$ ). The relative magnitudes of these compressional elastic constants are assigned as $C_{33}>C_{22}>C_{11}$ based on earlier Brillouin-scattering data. ${ }^{6}$ This result, however, is in conflict with the observation that phenothiazine is readily cleavable into $(00 n)$ platelets. Thus the compressive elastic constant perpendicular to the (001) plane, $C_{33}$, should be significantly smaller than those within the plane, $C_{22}$ and $C_{11}$. Lattice-dynamic calculations, based on atom-atom potentials, also describe a crystal that is more compliant (small elastic constant) along the $c$ axis rather than along the $a$ or $b$ axes of the unit cell. A significantly smaller phonon velocity was calculated for $q_{c}$ than for $q_{a}$ or $q_{b} \cdot{ }^{9}$

To date, five separate crystallographic investigations have been performed on phenothiazine; each has yielded a different, albeit not necessarily consistent, description of the crystal structure. An early x-ray diffraction description of the room-temperature crystal structure by Bell et al. ${ }^{10}$ was of a noncentrosymmetric monoclinic $P 2_{1}\left(C_{s}\right)$ structure. All other crystallographic studies,

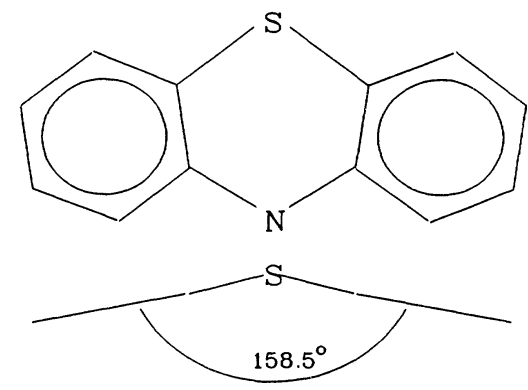

FIG. 1. Molecular structure of phenothiazine. 
however, describe lattices with inversion symmetry: a monoclinic $P 2_{1} / c \quad\left(C_{2 h}\right),{ }^{11}$ an orthorhombic Pnma $\left(D_{2 h}\right),{ }^{11,12}$ a polysynthetically twinned or disordered $P 2_{1} / c\left(C_{2 h}\right),{ }^{13}$ or a disordered $\operatorname{Pnma}\left(D_{2 h}\right)$ (Ref. 14) structure. The phenothiazine crystal grows with either a needle or platelike habit but polymorphic distinction is not clearly evident. ${ }^{13}$ Van de Waal and Feil, ${ }^{13}$ who first observed that phenothiazine undergoes a phase transition, believe that all the previous room-temperature structural studies are inaccurate because of molecular disorder along the $c$ axis and their structure reflects a correction for this. They also performed the only lowtemperature structural study, and concluded that the low-temperature lattice contained inversion symmetry. However, a weak pyroelectric effect observed in this study and by Wood, McCale, and Williams ${ }^{11}$ at $90 \mathrm{~K}$ would indicate a noncentrosymmetric lattice and a probable $P 2_{1}\left(C_{s}\right)$ low-temperature structure.

The formation of striae or domains suggests a change in lattice structure. In accord with Landau theory, a structural transition $P n m a\left(D_{2 h}\right)$ to $P 2_{1} / c\left(C_{2 h}\right)$, as previously proposed, should result from the spontaneous generation of an $e_{5}$ strain and an unstable $B_{2 u}$ acoustic mode. However, ambiguity concerning the lattice structure has resulted in a number of conflicting descriptions of the phase transition. Three specific mechanisms for the phase transition have emerged: order-disorder, ${ }^{13}$ overdamping of an optical libration, ${ }^{14,15}$ or a ferroelastic structural change.

In all cases but that of Sircar, Mishra, and Gupta, ${ }^{15}$ who assumed a $P 2_{1}\left(C_{s}\right)$ structure, there are reported experimental efforts to verify lattice type at room temperature. Nakayama and Ishii, ${ }^{2}$ in particular, regard the Raman-scattering tensor for phenothiazine as consistent with an orthorhombic lattice above $T_{c}$ and a monoclinic lattice below $T_{c}$. To obtain their result, they assume and require than an observed low-energy $22-\mathrm{cm}^{-1}$ optic mode be a molecular butterfly mode. As expected for a molecular mode, this mode displays no significant dependence on changes in temperature or pressure. However, a molecular assignment has yet to be confirmed. An optical mode at $49 \mathrm{~cm}^{-1}$ has been observed in the vaporphase Raman spectrum, ${ }^{7}$ but for a vibrational motion of this type, a shift to higher energy in the solid state would be expected because of the constraining intermolecular forces. Lattice-dynamic calculations also suggest that a $22-\mathrm{cm}^{-1}\left(B_{2 u}\right)$ vibration is acoustic. ${ }^{8}$ The calculations further suggest that if a butterfly mode does exist it would occur above $80 \mathrm{~cm}^{-1}$. The method of crystal growth also plays a significant role on the phase transition; $T_{c}$ 's are recorded near $220 \mathrm{~K}$ for solution grown samples ${ }^{13,15}$ and $240 \pm 2 \mathrm{~K}$ for vapor and boule grown crystals. ${ }^{1-3,6,16}$

Many of the conclusions drawn to date were necessitated by the experimental difficulties in obtaining sufficient data for a complete set of elastic constants in such lowsymmetry lattices. Because of calculational and experimental advances in our laboratory, we are able to report the results of Brillouin scattering involving 36 different acoustic-phonon modes of single crystals of phenothiazine, six of which are associated with the high-symmetry, true transverse, and true longitudinal wave vectors parallel to the $a$ and $b$ axes and three with wave vectors within $7^{\circ}$ of the high-symmetry $c$ axis. All measurements were made using a four-circle Brillouin spectometer ${ }^{17}$ and corrections were made for the temperature dependence and anisotropy of the optical indicatrix. In the previous Brillouin-scattering study, ${ }^{6}$ elastic constants were estimated solely from quasitransverse and quasilongitudinal phonon velocities.

The following sections present: (1) a brief description of the theory of Brillouin scattering and the determination of elastic constants with a four circle instrument, (2) a general method for determining the wave vector direction of an acoustic phonon, within an anisotropic crystal, coupled to externally non-normal incident or scattered laser radiation, (3) a detailed discussion of the crystallometry of very high-purity phenothiazine and the confusion surrounding the crystal structure, (4) a comparison of the results from our study with the theoretical latticedynamical results of Criado, ${ }^{9}$ the macroscopic properties of phenothiazine single crystals and the light-scattering and ultrasound results of Ecolivet et al. ${ }^{6}$ and Nakayama, and co-workers, ${ }^{1-3}$ and finally, (5) a model for the phase transition is proposed.

\section{BRILLOUIN SPECTROSCOPY: DETERMINATION OF ELASTIC CONSTANTS}

Brillouin scattering is treated extensively in the literature and the paper by Benedeck and Fritsch, ${ }^{18}$ and Fabelinskii, ${ }^{19}$ Cummins and Schoen, ${ }^{20}$ and Vacher and Boyer ${ }^{21}$ are particularly relevant. Only a brief summary is presented here.

The frequencies of acoustic phonons are obtained from an equation that describes the Doppler shift of Braggdiffracted light scattered from a sound wave traveling within a crystal at velocity $V(\hat{q})$ and of frequency $v_{\hat{q}}$. To account for optical anisotropy or birefringence, which is usually the case in organic crystal systems, dependence of the refractive indices of the incident and scattered rays, $n_{i}$ and $n_{s}$, respectively, is included. The resulting equation is:

$v_{s}=v_{0} \pm v_{q}=v_{0} \pm v_{0} \frac{V(\widehat{q})}{c}\left(n_{i}^{2}+n_{s}^{2}-2 n_{i} n_{s} \cos \theta\right)^{1 / 2}$,

where $\theta$ is the forward scattering angle and $v_{0}$ is the frequency of the incident light.

In the long-wavelength approximation, where the acoustic phonons are regarded as sound waves, polarizations and frequencies are related to the elastic constants $C_{\alpha \beta \gamma \delta}$ through the equation of motion

$$
\rho \ddot{U}_{\alpha}=C_{\alpha \beta \gamma \delta} \frac{\partial^{2} U_{\delta}}{\partial r_{\beta} \partial r_{\gamma}},
$$

where $U_{\alpha}$ is the set $\hat{u}$ of molecular displacements. This equation is entirely analogous to the vibrational equation of motion for molecules except now the displacements represent the long-wavelength, elastic continuum.

Using plane-wave solutions, Eq. (2) becomes 


$$
\rho V(\hat{q})^{2} U_{\alpha}=\Gamma_{\alpha \delta} U_{\delta},
$$

where $\rho$ is the volume density of the crystal, $V(\hat{q})$ is the phase velocity of the acoustic phonon and

$$
\Gamma_{\alpha \delta}=C_{\alpha \beta \gamma \delta} q_{\beta} q_{\gamma} .
$$

The matrix $\Gamma$ is symmetric with respect to the interchange of the dummy variables $\alpha$ and $\beta$, and $q_{\alpha}$ and $q_{\beta}$ are the direction cosines of the unit vector normal to the plane wave. Three mutually perpendicular directions of vibration are ascribed to a particular direction of propagation.

The frequencies and polarizations of acoustic waves are obtained from the standard secular equation:

$$
\operatorname{det}\left|\Gamma_{\alpha \delta}-\rho V(\widehat{q})^{2}\right|=0 .
$$

If the $\hat{q}$-dependent phonon eigenfrequencies and eigenvectors are known, the elastic constants can be determined. In low-symmetry crystals an analytical solution to Eq. (5) is only possible with pure vibrations, i.e., those vibrations that occur along directions of high symmetry within the crystal and that are perfectly transverse or longitudinal. Phonons in any other direction usually exhibit mixed characteristics of both. The propagation directions for pure transverse or pure longitudinal phonon modes can be determined ${ }^{22}$ and tables of them are available. $^{20}$ These directions are difficult, if not impossible, to experimentally access in low-symmetry molecular crystals.

The number of pure phonons dramatically decreases as the space-group symmetry is lowered. In such cases, unique solutions to Eqs. (3) and (5) become impractical. An analytical approach was developed to circumvent this problem through a least-squares minimization of Eq. (5) using reasonable initial approximations for either the elastic constants or a set of experimental phonon velocities from a sufficiently overdetermined number of propagation directions. ${ }^{23}$ The advantage of this method is that it eliminates the need to use purely longitudinal or purely transverse modes. Thus, modes of any arbitrary propagation direction $\hat{q}$ may be utilized and are generally referred to as quasilongitudinal or quasitransverse. The use of the quasimodes eliminates the need for destructive or damaging crystal cutting to obtain the desired faces.

For many low-symmetry crystal systems this method may be the only means to obtain the entire set of elastic constants. There are, however, some unavoidable inconveniences. One is that a large sampling of the different $\hat{q}$ vectors is required to give a good fit, and this requires many measurements. Crystal optics can also become increasingly complicated, especially for non-normal scattering. In addition, in the study of phase transitions and mode softening, it may still be important to observe certain pure modes because of possible strong spatial dispersion of $\hat{q}$, particularly along high-symmetry directions within the crystal. Ideally, one would like to have a spherical crystal to sample a statistically large number of directions. However, quite reasonable results can be obtained if efforts are made to include as many pure modes as possible and to use enough quasimodes to provide a reasonable statistical sampling of $\hat{q}$ within the crystal.

\section{EXPERIMENTAL}

\section{A. Purification, crystal growth, and crystallometry}

Phenothiazine is a pale, yellow solid obtained from the Aldrich Chemical Company at an approximate purity of 98\%. It was sublimed at least three times to remove inert material followed by repeated zone refining for further purification. ${ }^{24,25}$ Each of the five stages of the zone refiner used can be regulated to within $0.1^{\circ} \mathrm{C}$ for long periods of time to prevent excessive heating of the phenothiazine liquidus. Two boules were zone refined for approximately 100 and 200 passes. The resulting purified central section of the boule was off-white. The material was always handled in inert atmospheres.

High-quality single crystals of phenothiazine were subsequently grown by slow sublimation for eight weeks in a glass cell $7.5 \mathrm{~cm}$ in diameter and $2.5 \mathrm{~cm}$ wide. ${ }^{25}$ Both crystal habits of needles and platelets were observed. Although phenothiazine usually appears as platelets, a needle-shaped polymorph of phenothiazine was also reported by Bell et al. ${ }^{10}$ The needle-shaped crystals proved too fragile to handle. Therefore, all experiments were performed on the platelet samples. Typical crystal dimensions were $4 \times 3 \times 1.5 \mathrm{~mm}^{3}$.

Crystal interfacial angles were measured by optical goniometry. Utilizing the law of rational indices and the orthorhombic structure of McDowell, ${ }^{12}$ the differences between the experimental and calculated interfacial angles were less than $0.7 \%$. Similar agreement could not be obtained with the monoclinic structures of Bell et al. ${ }^{10}$ or van de Waal and Feil. ${ }^{13}$ Thus, the habit is consistent with an orthorhombic structure.

Single-crystal x-ray $\left(\mathrm{Cu}_{\alpha}\right)$ analysis was used to further verify the room-temperature axial assignments. Precession $\mathrm{x}$-ray patterns are consistent with an orthorhombic lattice (see Table I). Although the lattice appears orthorhombic from this measurement, the actual space group of the crystal should be regarded as somewhat inconclusive in light of previous, more extensive, $x$-ray studies. Furthermore, it was not possible to determine if the unit cell contains a center of inversion, although a slight pyroelectric effect was observed at $77 \mathrm{~K}$.

The refractive indices at various temperatures near and

TABLE I. Temperature-dependent refractive indices of phenothiazine at $514.5 \mathrm{~nm}$.

\begin{tabular}{cccc}
\hline \hline $\begin{array}{c}\text { Temperature } \\
(\mathbf{K})\end{array}$ & $n_{a}$ & $n_{b}$ & $n_{c}$ \\
\hline 295 & 1.70 & 2.00 & 1.79 \\
275 & 1.69 & 1.98 & 1.83 \\
265 & 1.72 & 1.93 & 1.77 \\
255 & 1.70 & 1.97 & 1.75 \\
250 & 1.71 & 1.97 & 1.75 \\
249 & 1.75 & 1.97 & 1.75 \\
248 & 1.68 & 1.95 & 1.73 \\
247 & 1.68 & 1.94 & 1.75 \\
245 & 1.67 & 1.94 & 1.76 \\
240 & 1.73 & 1.94 & 1.75 \\
220 & 1.79 & 1.99 & 1.75 \\
\hline \hline
\end{tabular}


through the phase transition were determined by normally incident specular reflection at the 514.5-nm scattering wavelength used in the Brillouin experiment. This temperature dependence of the refractive indices for the $a, b$, and $c$ axes of phenothiazine is given in Table $I$. Large changes are observed throughout the temperature range.

At least one-third of the vapor-grown crystals were observed to be macroscopically twinned at room temperature. The twinning occurred as an intergrowth within the larger crystal. Only untwinned crystals at $295 \mathrm{~K}$ were used for experiments.

All crystals of phenothiazine undergo a spontaneous, macroscopic twinning below $T_{c}$, and these lowtemperature twins form in domainlike structures throughout the crystal and result in visible striae. These twins appear unrelated to those observed at room temperature. The low-temperature twinning invariably occurred in a lamellar fashion with domain boundaries perpendicular to the $a$ axis (see Fig. 2) and resembled the polysynthetic twinning described by van de Waal and Feil, ${ }^{13}$ except on a macroscopic scale.

\section{B. Brillouin-scattering instrumentation}

The four-circle Brillouin spectrometer used in this experiment was constructed for rapid sampling of an unlimited number of propagation directions and is described elsewhere. ${ }^{17}$ Total instrument finesse is 78 ; theoretical finesse is $80 .^{26}$

A low-temperature apparatus, similar in principle to that used in four-circle, $x$-ray crystallography, bathed the crystal with a temperature controlled stream of cooled nitrogen with an outer concentric stream of dry, warm, nitrogen to prevent frosting. The device, following the

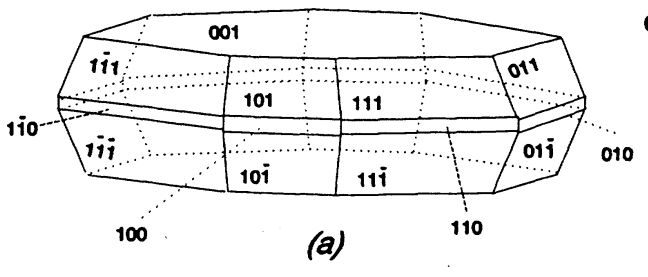

Cleavage Plane $=[001]$

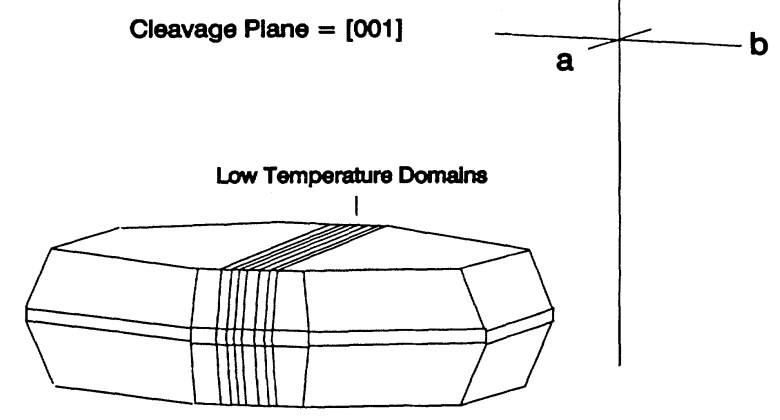

(b)

FIG. 2. Typical crystal morphology of phenothiazine showing crystallographic axes. A drawing of the crystal with the low-temperature domain structure is also presented. design of Rudman, ${ }^{27}$ allowed for the full advantage of the four-circle, Brillouin-scattering arrangement and did not interfere with the incident and scattering optical paths.

In determining the integrated intensities, all Brillouin peak shapes were corrected by removing the instrumental profile of the triple-pass, Fabry-Perot interferometer to arrive at phonon linewidths following a method of Lindsay, Brugess, and Shepherd. ${ }^{28}$

\section{Crystal optics}

Due to the strong birefringence of phenothiazine and the temperature dependence of the refractive indices, the actual position of the wave vectors of incident and scattered light, $\hat{k}_{i}$ and $\hat{k}_{s}$, respectively, within the crystal is of crucial importance. Their determination was treated in two ways depending upon the nature of the specific measurement.

The first is utilized for convenience where the complication of crystal optics can be avoided when $\hat{k}_{i}$ and $\hat{k}_{s}$ are both normal to crystal faces. The four-circle, scattering instrument permitted rapid positioning of the crystal to accommodate both normal incidence and normal scattering from naturally occurring faces. The polarization of the incident and scattered light can also be easily and accurately determined. Scattering from as many acoustic phonons as possible were measured this way.

The second case is the general method for determining the momentum vector. It is not always possible to use wave vectors of incident and scattered light normal to crystal facets because of the finite and usually small number of symmetrically inequivalent, naturally occurring faces. In organic crystals such as phenothiazine, the crystals are too fragile to cut without incurring significant sample damage or the possible introduction of a new phase. If $\hat{k}_{i}$ and $\hat{k}_{s}$ are non-normal to faces, crystal optics must be considered to determine propagation direction.

The determination of the momentum vector of light for arbitrary directions of incidence or scatter involves two independent constraints for a light wave propagating in a crystal. ${ }^{29}$ The first condition is expressed by Fresnel's equation which defines the inverse surface of wave normals. The second utilizes the continuity of the electric field across a boundary and the conservation of momentum. There are, in general, two wave vector solutions to these conditions commensurate with birefringence in an anisotropic crystal.

In practice, the determination of $\hat{q}_{i}$ and $\hat{q}_{s}$ can be very complicated and often requires iterative, graphic techniques only applicable under specific conditions. ${ }^{30}$ In this work, however, an approach was developed to determine the wave vectors through a weighted, nonlinear, leastsquares fitting procedure for both conditions described above. This analysis, which assumes plane polarized light, requires knowledge of vectors of $\hat{k}_{i}$ and $\hat{k}_{s}$, and of the optical indicatrix. The refractive indices that define the plane of incidence and scattering are determined along with the momentum vector of light within the crystal. The validity of this technique is quickly verified by using normally incident light where the solution is a vector normal to the boundary or face. 


\section{RESULTS}

\section{A. Heat of transition}

Differential scanning calorimetry (DSC) was used to determine the transition energy of the SPT for phenothiazine crystals grown by sublimation. A PerkinElmer model DSC-4 calorimeter was employed with the heat of freezing of water as the standard. The phenothiazine phase transition found at $248 \pm 0.5 \mathrm{~K}$ was endothermic and reversible with no annealing effects observed after three temperature cycles. The heat of transition for the vapor-grown crystals, $9.3 \mathrm{cal} / \mathrm{mol}$, was considerably smaller than the $47.8 \mathrm{cal} / \mathrm{mol}$ reported by Nakayama et al. ${ }^{1}$ who used a melt-grown specimen. This difference in the heat of transition is significant and probably reflects excess strain energy in the melt-grown sample. This is corroborated by the range of transition temperatures determined in this study, and by previous investigators, which supports a growth method dependence for the SPT.

\section{B. Temperature-dependent Brillouin spectra}

More than 1500 spectra were recorded for 12 different phonon wave vectors, or 36 different acoustic-phonon modes, at 11 successive temperatures through the phase transition. Each was determined for four different sets of polarizations of incident and scattered light: $V_{v}, V_{h}, H_{h}$, and $H_{v}$. The designations $V$ and $H$ refer to the polarization vectors of the light incident upon the crystal that are perpendicular and parallel, respectively (or nearly so, depending upon the optical indicatrix of the crystal) to the optical plane of the Brillouin instrument; subscripts $v$ and $h$ refer to polarizations of the scattered light defined in the same manner. At least two different free spectral ranges were recorded for each measurement to determine the order of the Brillouin components. Representative spectra are shown in Figs. 4 and 5. Each spectrum represents the sum of 25 scans; each scan represents the sum of photons scattered from the crystal observed for approximately $7 \mathrm{msec}$ per channel for a total of 1000 channels.

The plots in Fig. 3 display the temperature evolution of all three components of the Brillouin spectrum for the high-symmetry wave vectors along the $a, b$, and $c^{*}$ axes. The only way to access the true $c$-axis wave vector is in the backscattering arrangement from the large plate face of the crystal; $c^{*}$ is $7^{\circ}$ from the actual $c$ axis. Spectra were recorded at temperatures between 295 and $250 \mathrm{~K}$ but are not included for clarity and conservation of space. The spectra in Fig. 4 show the possible effect that domain formation may have on select Brillouin components.

\section{Elastic constants}

The complete set of elastic constants for phenothiazine was determined as described. Because of the uncertainty of the space-group assignment of phenothiazine at temperatures above and below $T_{c}$, two sets of elastic constants were calculated based on the orthorhombic and the monoclinic structures associated with phenothiazine.
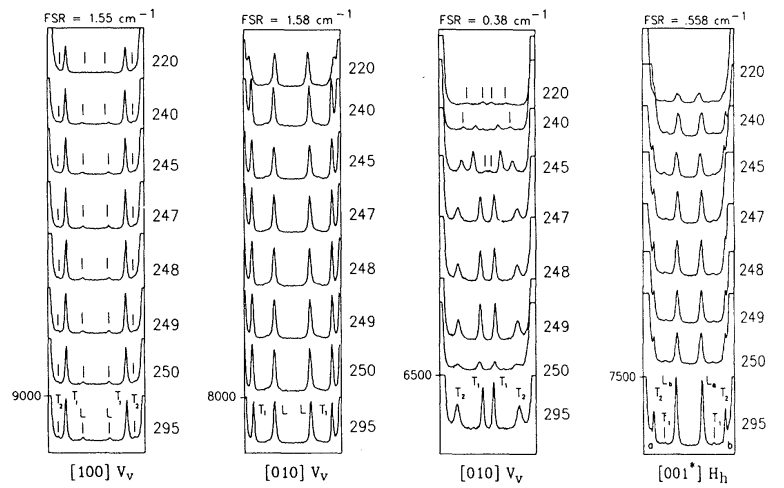

FIG. 3. Temperature evolution of the longitudinal $(L)$, transverse $\left(T_{1}\right)$, and transverse $\left(T_{2}\right)$ Brillouin spectral components for the high-symmetry wave vectors [100] and [010] and the wave vector $\left[001^{*}\right]$ which is within $7^{\circ}$ of the high-symmetry [001] wave vector. Absolute temperatures are shown on the right ordinates. The left ordinate shows the intensity as number of counts/sec. Abscissae show the free spectral range. Polarization configurations, defined in Sec. IV B are also indicated.
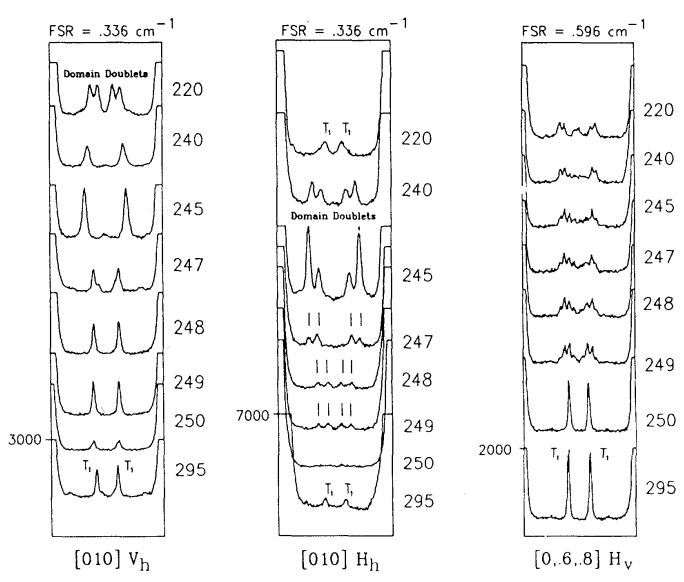

FIG. 4. Effect of structural domain formation on [010] and $[0,0.6 ., 0.8]$ phonon spectra for indicated polarization configurations (Sec. IV B). Absolute temperature (right ordinates), intensity in counts/sec (left ordinates) and free spectral ranges (abscissae) are displayed.

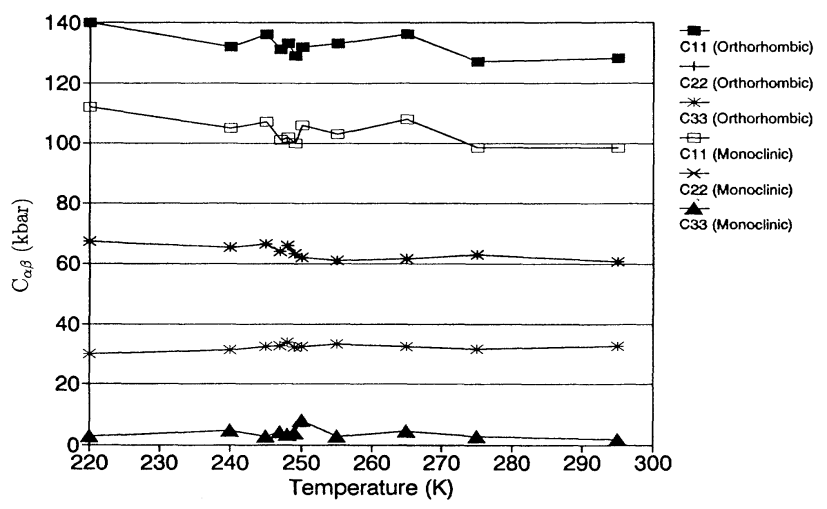

FIG. 5. Temperature evolution of the $C_{11}, C_{22}$, and $C_{33}$ elastic constants through $T_{c}$. 


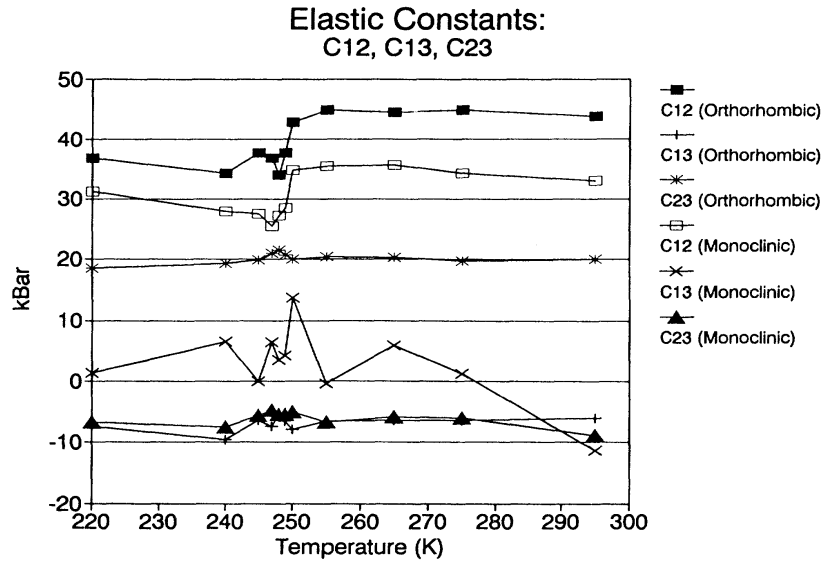

FIG. 6. Temperature evolution of the $C_{12}, C_{13}$, and $C_{23}$ elastic constants through $T_{c}$.

Acoustic-phonon wavevectors were determined using the relation of van de Waal and Feil ${ }^{13}(c>b>a)$

$$
a_{m} \simeq c_{0}, \quad b_{m} \simeq a_{0}, \quad c_{m} \simeq b_{0}+c_{0},
$$

which correlated the orthorhombic structure of McDowell $(b>c>a)$ to a monoclinic unit cell. Although the results of both the precession $x$-ray study and the optical goniometry support an orthorhombic, roomtemperature structure, the inclusion of the monoclinic set of elastic constants is presented because the structure of phenothiazine may actually be a polysynthetically twinned or disordered monoclinic structure or may become monoclinic at low temperature.

The refractive indices recorded at the same temperatures used in the Brillouin experiment were utilized in Eq. (5) to obtain the actual velocities of the acoustic phonons. In previous studies, a temperature-independent set of refractive indices obtained at room temperature was used. Using the actual values of the refractive indices at a given temperature has a significant impact on the elastic constant results. Errors in the experimentally deter-

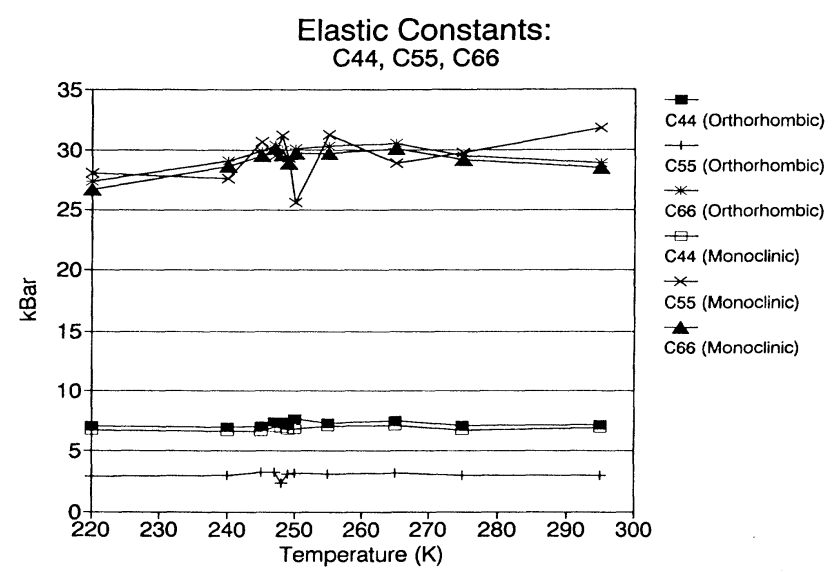

FIG. 7. Temperature evolution of the $C_{44}, C_{55}$, and $C_{66}$ elastic constants through $T_{c}$.

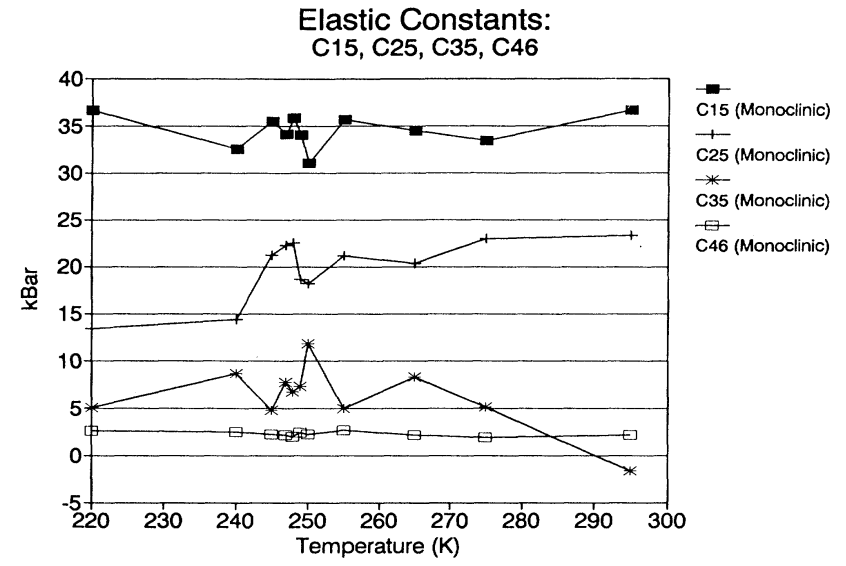

FIG. 8. Temperature evolution of the $C_{15}, C_{25}, C_{35}$, and $C_{46}$ elastic constants through $T_{c}$.

mined set of elastic constants ranging from 15 to $35 \%$ can result by ignoring the temperature dependence of the birefringence. The temperature dependence of the four sets of elastic constants is shown in Figs. 5-8.

\section{Intensity measurements of the Brillouin spectra}

A comparison in Fig. 9 of the room-temperature Brillouin intensities of the three longitudinal modes for phenothiazine traveling along the high symmetry axes $a$, $b$, and $c^{*}$, shows an interesting feature. The scattering intensity associated with the longitudinal phonon mode traveling along the $a$ axis is much smaller than that for the longitudinal phonons traveling along the $b$ or $c$ axes. This is shown by the ratios of the integrated intensities $I_{T} / I_{L}$ shown in Table II. These indicate disorder occurs along only the $a$ axis and perpendicular to the domain walls observed below $T_{c}$. The longitudinal and the quasilongitudinal modes typically display the greatest frequency shifts and intensities because the restoring forces and changes in dipole moment are generally larger for longitudinal than transverse phonon motions.

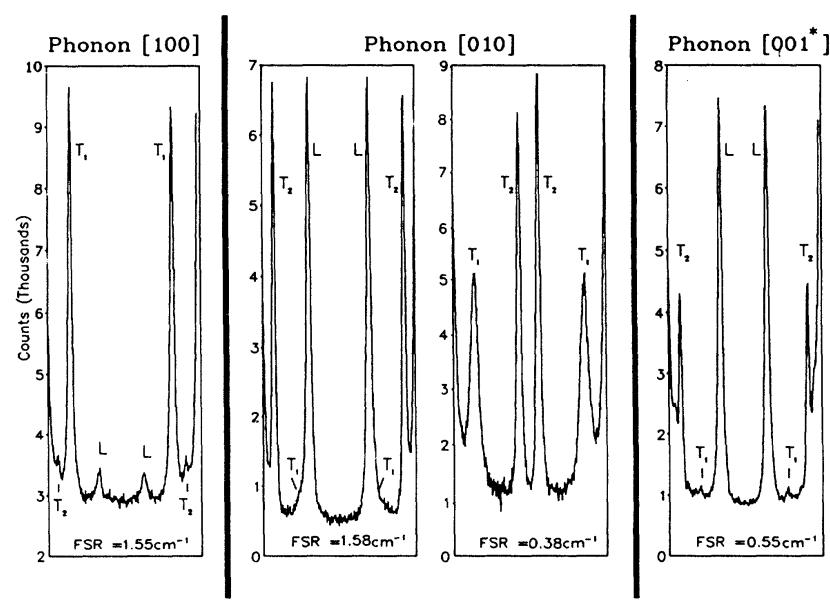

FIG. 9. Spectra of high-symmetry acoustic phonons assuming an orthorhombic lattice with $c>b>a$. 
TABLE II. Ratios of integrated intensities for pure acoustic modes of phenothiazine.

\begin{tabular}{ll}
\hline \hline Phonon & $I_{T} / I_{L}$ \\
\hline (100) $L$ & 1.0 \\
$(100) T_{1}$ & 8.5 \\
$(100) T_{2}$ & 0.14 \\
& \\
$(010) L$ & 1.0 \\
$(010) T_{1}$ & 0.90 \\
$(010) T_{2}$ & 0.42 \\
& \\
$(001) L$ & 1.0 \\
$(001) T_{1}$ & 0.02 \\
$(001) T_{2}$ & 0.07 \\
\hline \hline
\end{tabular}

\section{DISCUSSION}

\section{A. Role of twinning and domains}

The diffraction of laser light provides an important method of observing domain formation within single crystals of phenothiazine. Regular laser-light diffraction patterns were observed below $T_{c}$ when a laser beam of wavelength $514 \mathrm{~nm}$ was incident on the (011) and (111) faces. An illustration of the diffraction patterns appearing on a wall $2.5 \mathrm{~m}$ from the crystal is shown in Fig. 10.

The condition of interference of light is

$$
n \lambda=d[\sin (i)+\sin (r)],
$$

where $n$ is an integer, $\lambda$ is the wavelength of laser light, $d$ is the distance between domain walls, $i$ is the angle of incidence on a crystal face, and $r$ is the angle the refracted beam makes with the domain walls. Using Eq. (7), for the condition of laser light incident on the (011) face and from the measurement of the diffraction pattern on a wall, $d$ calculated to be approximately $1.5 \mu \mathrm{m}$. The presence of a regular diffraction pattern implies that the domains have remarkable regularity of size. Domain boundaries always formed parallel to the $a$ axis and were directly observed with a polarizing microscope to have dimensions that ranged from 1.0 to $3.0 \mu \mathrm{m}$.

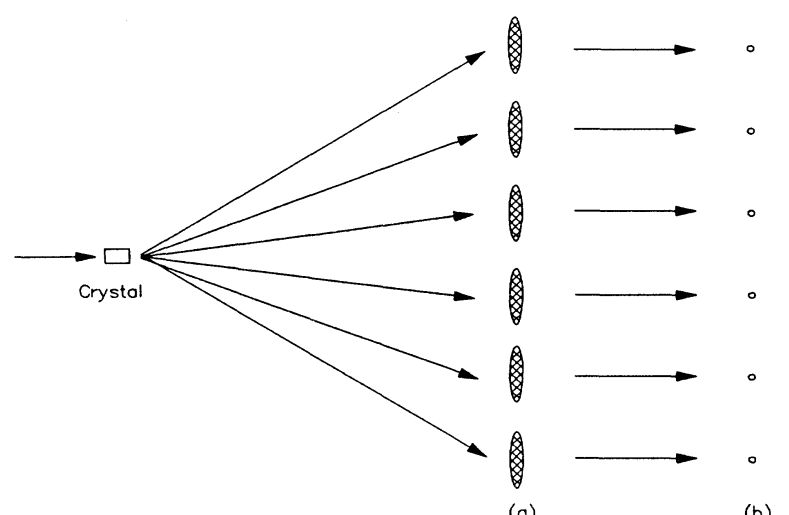

FIG. 10. Laser diffraction from the phenothiazine crystal at (a) room temperature and (b) below $T_{c}$.
The spacings of the diffracted light beams do not change with temperature within the 220-295 K range. The size of the diffracted spot (see Fig. 10) varies significantly with temperature, but decreases in size as the temperature is lowered. This is indicative of the degree of increasing translational order with decreasing temperature. This supports the conjecture by van de Waal and Feil of polysynthetic twinning in the roomtemperature lattice.

The effect on the monochromatic light scattered from a crystal with a mosaic of domains is to broaden the band shape and reduce the intensity. ${ }^{31-33}$ Thus, as the number of translationally inequivalent lattices increases within a scattering volume, the intensity of the inelastically scattered light decreases. Twinning and domain formation limit the extent of long-range ordering, effectively reducing the size of the lattice the light would sample. This is particularly clear in the spectrum of the [100] $V_{h}$ Brillouin transverse $T_{1}$ component, which significantly decreases in intensity as as domain walls became visible (see Fig. 5).

The low intensity of the longitudinal [100] Brillouin component at room temperature suggests that the twinning is present well above the phase-transition temperature. This is consistent with $\mathrm{x}$-ray results that show the atomic displacement parameters for phenothiazine are largest along the $c$ axis of the crystal. This also supports the possibility of polysynthetic twinning with domain walls perpendicular to this axis.

A similar reduction in intensity can also occur through an overdamped lattice vibration. An optical mode of high amplitude was reported in the NMR experiment of Sircar, Mishra, and Gupta where the lattice motion was observed to dampen significantly as the temperature was reduced. In Fig. 5 it can be seen that the transverse $T_{1}$ Brillouin component [010] $H_{h}$ increases significantly in intensity as the temperature is reduced. It is not yet clear if this acoustic mode is coupled to the overdamped optical mode. It is likely that both effects, domain disorder and the high amplitude motion, are linked, and an experiment which exploits the uniaxial nature of the disorder would provide a great deal of information.

\section{B. The acoustic modes and the SPT}

Lattice symmetry has a direct effect on Brillouinscattering tensors and phonon velocities. Table III displays the symmetrically equivalent phonon modes in an orthorhombic lattice. In this experiment the velocities of phonons are consistent with an orthorhombic lattice except for one set. The velocities of the two modes which relate to the stiffness of the $e_{5}$ shear strain differ significantly: $348.0 \mathrm{~m} / \mathrm{sec}$ (within $7^{\circ}$ of the true wave vector) versus $1133.0 \mathrm{~m} / \mathrm{sec}$ (true). These two modes are obviously not symmetrically related unless there is a significant amount of spatial dispersion in the former mode. The lattice, therefore, does not display all the orthorhombic symmetry operations as a result of uniaxial disorder or the lattice is actually disordered monoclinic (quasiorthorhombic). The agreement between the experi- 
TABLE III. Summary of experimental acoustic-phonon velocities from various studies. Symmetrically equivalent phonon modes designated by letter (orthorhombic lattice only).

\begin{tabular}{|c|c|c|c|c|}
\hline$\hat{q}$ & $\hat{e}$ & $\begin{array}{c}\text { Criado } \\
\text { (Ref. 9) } \\
\text { km/s }\end{array}$ & $\begin{array}{l}\text { This work } \\
\mathrm{km} / \mathrm{s}\end{array}$ & $\begin{array}{c}\text { Ecolivet et al. } \\
\text { (Ref. 6) } \\
\mathrm{km} / \mathrm{s}\end{array}$ \\
\hline$[100]$ & {$[100]$} & 3.44 & 3.10 & 2.44 \\
\hline$d[100]$ & {$[010]$} & 2.04 & 1.73 & 1.97 \\
\hline$e \quad[100]$ & [001] & 1.11 & 1.13 & 0.430 \\
\hline [010] & {$[010]$} & 2.36 & 2.12 & 2.56 \\
\hline$d[010]$ & {$[100]$} & 1.54 & 1.52 & 1.36 \\
\hline$f \quad[010]$ & {$[001]$} & 0.789 & 0.780 & 1.97 \\
\hline [001] & {$[001]$} & 1.25 & 1.64 & 3.41 \\
\hline$e \quad[001]$ & {$[100]$} & 0.433 & 0.619 & 0.430 \\
\hline$f \quad[001]$ & [010] & 0.382 & 0.348 & 1.36 \\
\hline
\end{tabular}

mental velocities obtained in this work and those calculated by Criado $^{9}$ for a monoclinic lattice are shown in Table III. The velocities estimated from Ecolivet's et al. ${ }^{6}$ set of elastic constants are listed for comparison.

Although the Brillouin spectra in our study exhibited significant dynamic behavior, including polarization breakthrough and multiplet formation from domains, the frequencies of the acoustic modes displayed no anomalous temperature dependence. No softening was observed for any acoustic mode. The frequencies of the two acoustic modes $\{\hat{q}=[100], \hat{e}=[001]\}$ or $\{\hat{q}=[001], \hat{e}=[100]\}$ (equivalent in an orthorhombic lattice) should have decreased to zero frequency at $T_{c}$ to be consistent with the ultrasound results of Nakayama, Ishii, and Sawada. ${ }^{3}$

\section{Elastic constants}

The room-temperature elastic constants in Table IV determined by various investigators and experiments are shown for comparison. Those listed for Criado ${ }^{9}$ were calculated from velocities extracted from his published dispersion curves based on his lattice-dynamical calculations.

There are significant differences for values of the $C_{33}$ elastic constant which describes the stiffness along the $c$ axis; $C_{33}\left[C_{33}=C_{c c}\right]$ is the largest for Nakayama, Ishii, and Sawada ${ }^{3}$ and Ecolivet et al.$^{6}$ and the smallest for this work and those determined from the calculated phonon velocities of Criado. ${ }^{9}$ Confusion in axial labeling of the lattice is not possible because all investigators refer to the $x$ axis as being parallel to the long axis of the phenothiazine molecule. It is possible, however, that molecular or other unit-cell axes were not otherwise correctly determined. The use of twinned specimens also would cause assignment errors.

The morphological differences between a melt-grown and a vapor-grown crystal of phenothiazine are substantial so that a comparison cannot be made between the two. It is generally accepted that melt-grown crystals have higher defect concentrations than vapor-grown samples. Defects in crystals tend to make them harder, and therefore, melt-grown samples are less compressible than vapor-grown samples. Strains generated by the meltgrowth process may obscure a spontaneously generated strain or they might appear as a strain instability unrelated to the SPT, e.g., annealing effects. In addition, a large dispersion near $\hat{q}=0$ may exist. $\mathrm{Rb}_{2} \mathrm{ZnCl}_{4}$, for example, displays a significant dispersion between the ultrasound and Brillouin measurements of the $C_{33}$ elastic constant. ${ }^{34,35}$

The nearly complete disagreement with the Brillouinscattering study of Ecolivet et al. ${ }^{6}$ is more problematic. They appear to have used vapor-grown crystals and sampled a large number of $\hat{q}$ vectors, but the inconsistency of their $C_{33}$ value with respect to the macroscopically ob-

TABLE IV. Comparison of elastic constants in kbars from various studies of phenothiazine.

\begin{tabular}{|c|c|c|c|c|c|c|c|}
\hline & $\begin{array}{l}\text { Nakayama, Ishii, } \\
\text { and Sawada (Ref. 3) }\end{array}$ & $\begin{array}{c}\text { Ecolivet } \\
\text { et al. (Ref. 6) }\end{array}$ & $\begin{array}{l}\text { Criado } \\
\text { (Ref. 9) }\end{array}$ & This work & This work & This work & This work \\
\hline $\begin{array}{l}\text { Space } \\
\text { group }\end{array}$ & Ortho. & Ortho. & $\begin{array}{c}\text { Monoclinic } \\
\beta=92.6\end{array}$ & Ortho. & $\begin{array}{c}\text { Monoclinic } \\
\beta=92.6\end{array}$ & $\begin{array}{l}\text { Monoclinic } \\
\beta=105.67\end{array}$ & $\begin{array}{c}\text { Monoclinic } \\
\beta=110.87\end{array}$ \\
\hline Method & Ultrasound & Brillouin & Calculated & Brillouin & Brillouin & Brillouin & Brillouin \\
\hline \multicolumn{8}{|l|}{$\begin{array}{l}\text { Elastic } \\
\text { constants }\end{array}$} \\
\hline$C_{11}$ & & 80.9 & 159.0 & 128.0 & 125.0 & 98.7 & 92.2 \\
\hline$C_{12}$ & & 59.7 & & 43.8 & 43.5 & 33.0 & 30.5 \\
\hline$C_{13}$ & & 2.61 & & 0.126 & 11.8 & -11.3 & -0.959 \\
\hline$C_{15}$ & & & & & 13.8 & 36.6 & 36.6 \\
\hline$C_{22}$ & & 88.7 & 75.5 & 60.8 & 60.8 & 60.8 & 60.8 \\
\hline$C_{23}$ & & 44.6 & & -34.5 & -17.7 & -8.86 & -5.52 \\
\hline$C_{25}$ & & & & & 5.34 & 23.3 & 24.9 \\
\hline$C_{33}$ & & 157.6 & 21.2 & 33.0 & 21.2 & 1.91 & 2.53 \\
\hline$C_{35}$ & 150.0 & & & & 14.7 & -1.60 & 4.75 \\
\hline$C_{44}$ & & 25.2 & 3.90 & 7.23 & 6.84 & 6.91 & 6.91 \\
\hline$C_{46}$ & & & & & -1.88 & 2.15 & 2.21 \\
\hline$C_{55}$ & & 2.50 & 3.54 & 2.96 & 13.4 & 31.8 & 33.3 \\
\hline$C_{66}$ & 2.0 & 52.8 & 40.9 & 28.9 & 28.3 & 28.5 & 28.5 \\
\hline
\end{tabular}


servable [001] cleavage suggests a misassignment of the crystallographic axis with respect to the crystal habit. Further, they were not able to account for crystal optics in the light-scattering experiments and they give no indication of an awareness of twinned samples. It is not possible to reassign their axes to redetermine elastic constant values from their data because the direct observation of high-symmetry, acoustic modes was not reported. Results based almost entirely on quasitransverse or quasilongitudinal modes admit an extremely large number of propagation directions and obviate a unique solution.

Ecolivet et al. ${ }^{6}$ have assumed that the largest elastic constant of phenothiazine should be along the longest molecular axis based on problematical isostructural arguments from previous elastic constant studies of the polyacenes, anthracene, and naphthalene. The molecular long axis is also the $c$ axis in these acene crystals, but the unit cell of phenothiazine is not in any way isostructural with anthracene or naphthalene. In anthracene and naphthalene, the molecules directly abut one another along the long axis of the molecules. This is not so with phenothiazine; layers of molecules in successive $(00 n)$ planes do not pack immediately over one another with abutting ends in a face-centered fashion thereby leaving an intermolecular gap of one unit-cell length perpendicular to the $c$ axis. If phenothiazine were to be compressed along the $c$ axis, the molecules could more easily slip past each other, encountering less interference in this direction. Also, the phenothiazine molecule itself is expected to be more conformationally labile than anthracene and naphthalene, since it may bend in a butterfly fashion along the $N-S$ axis. This would further increase compressibility of the crystal along the $c$ axis.

\section{Temperature dependence of elastic constants}

Acoustic-phonon measurements and the subsequent determination of unstable strains may be significantly affected as the crystal delaminates into $(00 n)$ platelets with changes in temperature and pressure. Strains generated through delamination or by a changing structure may be undiscernable. Further, significant differences in inherent strain of the crystal due to intermolecular interaction and strains generated by the crystal-growth process (i.e., differences in defect concentration) may exist because the single crystals of phenothiazine are highly compliant. The observed hysteretic behavior of the lowtemperature domains of phenothiazine may be due to higher-order strain effects such as ferrobielasticity.

It is apparent from Figs. 5 through 8 that the elastic stiffness of the phenothiazine single crystal undergoes significant but complex changes through the phase transition. Instabilities in the elastic constants $C_{55}$ and $C_{33}$ have been implicated in the phase transition. The former is associated with a shear in the $a c$ plane and $C_{55} \rightarrow 0$ as $T \rightarrow T_{c}$ would correspond directly to a transition from $P 2_{1} / c\left(C_{2 h}\right)$ to $P n m a\left(D_{2 h}\right)$. In this work, $C_{55}$ displayed no significant change through the phase transition if the room-temperature lattice is considered to be orthorhombic. The remaining elastic constants, $C_{44}$ and $C_{66}$, that could be associated with a change in lattice symmetry, displayed only slight changes determined for any of the four lattice types.

A summary of the behavior exhibited by the complete set of elastic constants obtained in this study suggests an elastic fluctuation near $T_{c}$ but no significant, symmetrybreaking, structural instabilities. Among the compressional elastic constants, $C_{11}$ and $C_{22}$ show the greatest fluctuation through $T_{c}$ for both lattice structures. As the temperature is lowered, there appears to be an expansion along the $b$ axis and a compression along the $a$ axis as indicated by $C_{22}$ and $C_{11}$, respectively. Below $T_{c}$, both tend to increase as the temperature is decreased, as would be expected for a contracting lattice. The elastic constant $C_{33}$ remains essentially constant throughout the temperature range for both structures.

Of the off-diagonal compressional elements, only $C_{12}$ exhibits any temperature dependence near $T_{c} . C_{13}$ displays a significant change but only if the lattice is considered to be monoclinic $\left(\beta=105.67^{\circ}\right) . C_{23}$ remains constant throughout the range, but the signs are different in the orthorhombic and monoclinic structures. Of the rest of the elastic constants, the most significant changes occur in the off-diagonal $C_{15}$ element and the more dominant changes in $C_{25}$ and $C_{35}$ only occur if the elastic constants are calculated for monoclinic lattice. Finally, $C_{46}$, which connects a $b c$ shear under the action of an $a b$ shear (or vice versa), does change slightly through the phase transition, but the most significant change for $C_{46}$ arises from the lattice choice.

It should be noted that of the temperature-dependent elastic constants only the orthorhombic set yields a positive energy density throughout the entire temperature range. This is significant because the orthorhombic lattice has been determined to be the least stable of the three possible structures associated with phenothiazine$\operatorname{Pnma}\left(D_{2 h}\right), P 2_{1} / c\left(C_{2 h}\right)$, or $P 2_{1}\left(C_{s}\right)$-based on lattice energy calculations. ${ }^{9}$ A negative energy density does not signify an unrealistic result, since phenothiazine forms domains to achieve stability. ${ }^{36}$ A negative energy density may also imply a metastable phase of the crystal phase above $T_{c}$ and a trend toward stability at low temperature.

\section{E. Ferroelasticity and the SPT of phenothiazine}

A proper, ferroelastic, phase transition is unlikely, since the $e_{5}$ strain is apparently not the primary order parameter. An unstable $\hat{q} \sim 0$ acoustic motion is not the principal driving force behind the phase transition in phenothiazine unless the transition is antiferroic. If the phase transition of phenothiazine occurs as disordered monoclinic $P 2_{1} / c\left(C_{2 h}\right)$ to monoclinic $P 2_{1}\left(C_{s}\right)$, the unit cell would be halved and the Brillouin zone of the lowtemperature phase would be doubled. In this case, the order parameter would be expected to correspond to an acoustic motion at the Brillouin-zone boundary of the high-temperature phase and the low-temperature monoclinic structure of phenothiazine would not contain a center of inversion. The observation of the pyroelectric effect in this work and by Wood, McCale, and Williams ${ }^{11}$ supports a $P 2_{1}\left(C_{s}\right)$ low-temperature structure which is 


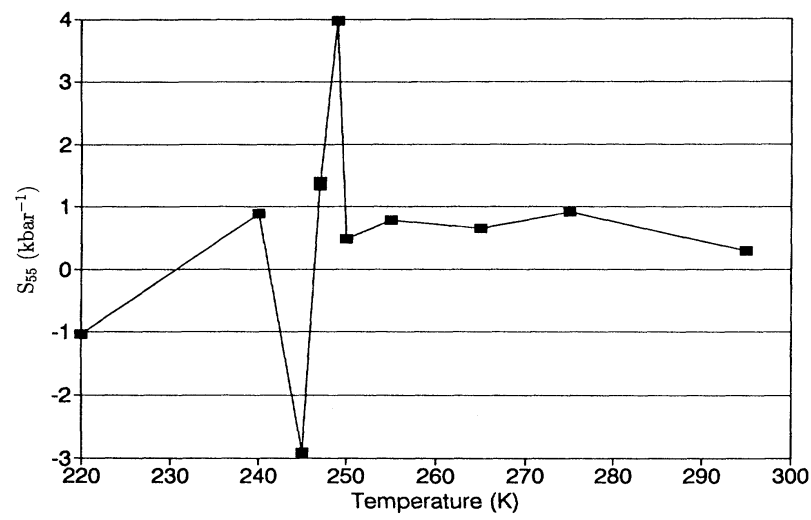

FIG. 11. Temperature dependence of the $s_{35}$ compliance element.

more stable than the $P 2_{1} / c\left(C_{2 h}\right)$ lattice based on lattice static calculations. ${ }^{37}$ Thus, the phase transition may be antiferroic.

To date, no evidence of cell doubling has been recorded for phenothiazine below $T_{c}$. Nevertheless, this does not preclude the possibility because anomalous diffraction effects may be introduced by polysynthetic twinning, disorder or domain formation.

The Raman-active lattice modes of phenothiazine have been observed to show significant anomalies under both changes in temperature and pressure. Thus the possibility of an improper ferroelastic SPT cannot be ruled out and the importance of the unstable optical modes to the SPT in phenothiazine requires further study.

\section{F. Ferrobielasticity}

Another explanation may be advanced, however. Phenothiazine may be ferrobielastic. The $s_{35}$ element of the compressibility tensor displays a significant temperature dependence through the phase transition. This is important, since it could make a ferrobielastic crystal appear ferroelastic at low temperature due to an induced $e_{5}$ strain. In phenothiazine, the macroscopically observed domains appear to change under $a$ - or $b$-axis uniaxial stresses. An induced $e_{5}$ strain could be generated along with other purely compressional strains if the compressibility coefficient $s_{35}$ induces different responses in twins. Domain switching would then be observed. The temperature-dependent values of $s_{35}$, determined for $\beta=105.67^{\circ}$, are displayed in Fig. 11 . It is evident that this element shows an anomaly through the phase transition. Thus, a ferrobielastic response of phenothiazine would explain the pressure sensitive domain behavior observed by Nakayama et al. ${ }^{1}$ and the lack of temperature-dependent behavior of the $C_{55}$ elastic constant found in this study.

\section{CONCLUSION}

The specific identity of the lattice structure and the nature of the structural phase transition in phenothiazine have historically generated conflicting conclusions. The single crystals exhibit a number of complicating features which make evaluation of lattice vibrations difficult: uniaxial disorder, macroscopic and polysynthetic twinning, structural domains, structural hysteresis, a strong cleavage plane, and plasticity. These features are a direct manifestation of the complex dynamic behavior of the crystal and this study integrates them into a coherent picture of the anharmonicity of the $\hat{q} \sim 0$ acoustic modes in relation to the phase transition.

The results presented here are consistent with a unaxially disordered crystal which undergoes an order-disorder phase transition. Structural domains are found to be present above and below $T_{c}$; however, room-temperature domain boundaries are blurred by uniaxial disorder. ${ }^{13}$ As samples of phenothiazine are cooled below $T_{c}$, domain walls become visible and cause the multiplet formation observed in the Brillouin spectra. Although it has been postulated that the phase transition of phenothiazine is from disordered $P 2_{1} / c\left(C_{2 h}\right)$ to ordered $P 2_{1} / n\left(C_{2 h}\right)$ (Ref. 13) or from $P n m a\left(D_{2 h}\right)$ to $P 2_{1} / n\left(C_{2 h}\right)$, this research suggests that the actual structural change is from disordered Pnma $\left(D_{2 h}\right)$ [or disordered $\left.P 2_{1} / c\left(C_{2 h}\right)\right]$ to ordered $P 2_{1}\left(C_{s}\right)$ (Ref. 14) if the observation of the pyroelectric effect at low temperature is correct. This assignment is based on the behavior of the elastic constants and the observation of a weak pyroelectric effect below $T_{c}$.

Inconsistencies in previous studies with the macroscopic properties and a possible misassignment of lattice axes bring the previously conjectured ferroelastic assignment into doubt. Reports of mode softening in the ultrasound experiment on melt-grown samples could arise from annealing effects, crystal delamination and domain formation. The observed domain switching within phenothiazine is probably due to the $s_{35}$ compliance rather than the $e_{5}$ strain.

The examination of both the high symmetry and quasilongitudinal or quasitransverse acoustic modes in this study show that phenothiazine exhibits a higher-order effect such as ferrobielasticity below $T_{c}$. Instabilities in elements of the elastic or compliance tensor, rather than the strains, may still play a primary role in the phase transition. NMR studies have strongly supported a phase transition governed by an unstable optical mode or an optical mode which is strongly coupled to the lattice. $^{8,15}$ In addition, recent lattice-dynamical calculations indicate an important role for acoustic-optical mode coupling in the phase transition. ${ }^{38}$ It is, therefore, possible that the elastic or compliance elements that are unstable through the phase transition and that are of the appropriate symmetry are coupled to the observed anharmonic optical modes. 
${ }^{*}$ Present address: Harris Laboratories, 624 Peach Street, Lincoln, NE 68501.

${ }^{\dagger}$ Author to whom correspondence should be addressed.

${ }^{1}$ H. Nakayama, K. Ishii, E. Chijiwa, M. Wada, and A. Sawada, Solid State Commun. 55, 59 (1985).

${ }^{2}$ H. Nakayama and K. Ishii, Chem. Phys. 114, 431 (1987).

${ }^{3}$ H. Nakayama, K. Ishii, and A. Sawada, Solid State Commun. 67, 179 (1988).

${ }^{4}$ V. K. Wadhawan, Phase Trans. 3, 3 (1982).

${ }^{5}$ G. Araki, M. Mukai, H. Nakayama, and K. Ishii, Bull. Chem. Soc. Jpn. 63, 897 (1990).

${ }^{6}$ C. Ecolivet, M. Sanquer, K. Ishii, and H. Nakayama, Phys. Rev. B 44, 4185 (1991).

${ }^{7}$ H. Nakayama, M. Mukai, R. Hagiwara, and K. Ishii, J. Phys. Chem. 94, 4343 (1990).

${ }^{8}$ S. Ishimaru, N. Nakamuro, and H. Chihara, J. Phys. Soc. Jpn. 60, 2424 (1991).

${ }^{9}$ A. Criado, Solid State Commun. 76, 61 (1990).

${ }^{10}$ J. D. Bell, J. F. Blount, O. V. Briscoe, and H. C. Freeman, Chem. Commun. 1656 (1968).

${ }^{11}$ R. G. Wood, C. H. McCale, and G. Williams, Philos. Mag. 31, 71 (1941).

12J. J. H. McDowell, Acta Crystallogr. B 32, 5 (1976).

${ }^{13}$ B. W. van de Waal and D. Feil, Acta Crystallogr. B 33, 314 (1977).

${ }^{14}$ R. D. Stigler, Ph.D. thesis, Universität Stuttgart, 1988.

${ }^{15}$ R. Sircar, S. C. Mishra, and R. C. Gupta, Indian J. Appl. Phys. 18, 757 (1980).

${ }^{16}$ N. Karl and H. Rommel (private communication).

${ }^{17}$ R. Dye, J. Sartwell, and C. J. Eckhardt, Rev. Sci. Instrum. 60, 2610 (1989).

${ }^{18}$ G. B. Benedek and K. Fritsch, Phys. Rev. 149, 149 (1966).

${ }^{19}$ I. L. Fabelinskii, Molecular Scattering of Light (Plenum, New York, 1968).
${ }^{20}$ H. Z. Cummins and P. E. Schoen, in Laser Handbook, edited by F. T. Arecchi and E. O. Schulz-Dubois (North-Holland, Amsterdam, 1972), Chap. E1.

${ }^{21}$ R. Vacher and L. Boyer, Phys. Rev. B 6, 283 (1972).

${ }^{22}$ K. Brugger, J. Appl. Phys. 36, 759 (1965).

${ }^{23}$ K. H. Brose, Ph. D. thesis, University of Nebraska, 1988.

${ }^{24}$ T. E. Anderson, Ph.D. thesis, University of Nebraska, 1985.

${ }^{25}$ N. Karl, in Crystais: Growth, Properties, and Applications, edited by H. C. Freyhardt (Springer, Berlin, 1980), Vol. 4, p. 1.

${ }^{26}$ G. Hernandez, Fabry-Perot Interferometers (Cambridge University Press, Cambridge, 1986).

${ }^{27}$ R. Rudman, Low-Temperature X-ray Diffraction (Plenum, New York, 1976).

${ }^{28}$ S. M. Lindsay, S. Burgess, and I. W. Shepherd, Appl. Opt. 16, 1404 (1977).

${ }^{29}$ M. Born and E. Wolf, Principles of Optics (Pergamon, Oxford, 1970).

${ }^{30}$ F. D. Bloss, An Introduction to the Methods of Optical Crystallography (Holt, Rinehart and Winston, New York, 1961).

${ }^{31}$ M. Leontowitsch and S. Mandelstam, Jr., Phys. Z. Sowjet Union 1, 317 (1931).

${ }^{32}$ M. Leontowitsch and S. Mandelstam, Jr., Z. Phys. 75, 350 (1932).

${ }^{33}$ M. Born and K. Huang, Dynamical Theory of Lattices (Clarendon, Oxford, 1954).

${ }^{34}$ A. Yamanaka, M. Kasahara, and I. Tatsuzaki, J. Phys. Soc. Jpn. 50, 735 (1981).

${ }^{35}$ Y. Luspin, M. Chabin, G. Hauret, and F. Gilletta, J. Phys. C 15, 1581 (1982).

${ }^{36} \mathrm{M}$. Born and K. Huang, Dynamical Theory of Lattices (Oxford University Press, Oxford, 1954).

${ }^{37}$ J. Sartwell and C. J. Eckhardt (unpublished).

${ }^{38}$ A. Criado and T. Luty, J. Phys. Soc. Jpn. 61, 2835 (1992). 